\title{
Erratum: Logarithmic accuracy of parton showers: a fixed-order study
}

\author{
Mrinal Dasgupta, ${ }^{a}$ Frédéric A. Dreyer, ${ }^{b}$ Keith Hamilton, ${ }^{c, d}$ Pier Francesco Monni ${ }^{d}$ \\ and Gavin P. Salam ${ }^{d, 1}$ \\ ${ }^{a}$ Consortium for Fundamental Physics, School of Physics and Astronomy, \\ University of Manchester, \\ Manchester M13 9PL, U.K. \\ ${ }^{b}$ Center for Theoretical Physics, Massachusetts Institute of Technology, \\ Cambridge, MA 02139, U.S.A. \\ ${ }^{c}$ Department of Physics and Astronomy, University College London, \\ London, WC1E 6BT, U.K. \\ 'Theoretical Physics Department, CERN, \\ CH-1211 Geneva 23, Switzerland \\ E-mail: Mrinal.Dasgupta@manchester.ac.uk, fdreyer@mit.edu, \\ keith.hamilton@ucl.ac.uk, pier.monni@cern.ch, gavin.salam@cern.ch
}

ERratum TO: JHEP09(2018)033

ABSTRACT: We detail corrections to the $\alpha_{s}^{3} L^{3}$ results shown in table 1 of ref. [1]. The conclusions are unchanged.

ArXiv EPrint: 1805.09327

In ref. [1], table 1 intended to show results for $\delta \ln \Sigma$, while appendix B outlined how to obtain results for $\delta \Sigma$. An incorrect assumption was made in relating the two, which impacted the third order results, i.e. those for the vector $p_{t}$ sum and the thrust. The new results are shown in table 1 of this Erratum. The second-order results are unchanged.

The vector $p_{t}$ result has been obtained semi-analytically and verified numerically. Only the thrust relies on a full numerical evaluation. Within the approach of appendix B, the only effects that we evaluate are those due to the recoil prescription, which set in from second order. Writing the effect of the recoil prescription on $\Sigma_{n}$ as $\delta \Sigma_{n}$, and exploiting $\delta \Sigma_{1}=0$, we have $\delta \ln \Sigma=\alpha_{s}^{2} \delta \Sigma_{2}+\alpha_{s}^{3}\left(\delta \Sigma_{3}-\Sigma_{1} \delta \Sigma_{2}\right)+\mathcal{O}\left(\alpha_{s}^{4}\right)$ and directly evaluate the third order term. That evaluation is shown in figure 1 together with the result of a logarithmic fit.

\footnotetext{
${ }^{1}$ On leave from CNRS, UMR 7589, LPTHE, F-75005, Paris, France and from Rudolf Peierls Centre for Theoretical Physics, 1 Keble Road, Oxford OX1 3NP, U.K. .
} 


\begin{tabular}{|cc|}
\hline Observable & $\mathrm{NLL}_{\ln \Sigma}$ discrepancy \\
\hline vector $p_{t}$ sum & $-0.250 \bar{\alpha}^{3} L^{3}$ \\
\hline $1-T$ & $+0.016_{-0.001}^{+0.001} \bar{\alpha}^{3} L^{3}$ \\
\hline
\end{tabular}

Table 1. Corrected NLL differences between the Pythia and Dire shower-algorithm results and the analytic resummation for different observables, at the first non-trivial perturbative order in $\bar{\alpha} L=$ $2 C_{F} \alpha_{s} L / \pi$, with $L=\ln 1 / v$, for the thrust and vector $p_{t}$ sum. The uncertainty in the thrust case corresponds to a statistical integration error, while the vector $p_{t}$ sum has obtained semi-analytically. Note that the thrust result has been obtained specifically within the approximation discussed in appendix B of ref. [1]. Our subsequent detailed analysis of the thrust in dipole-type showers has revealed a non-trivial interplay between $\mathrm{NNLL}_{\ln \Sigma}$ logarithmic terms and factors $\left(\alpha_{s} L^{2}\right)^{n}$, with subtleties related to the breaking of standard exponentiation. The nature of this interplay with the full shower evaluation of $\mathrm{NNLL}_{\ln \Sigma}$ terms (rather than just the recoil considerations of appendix B) goes beyond the scope of the original article.

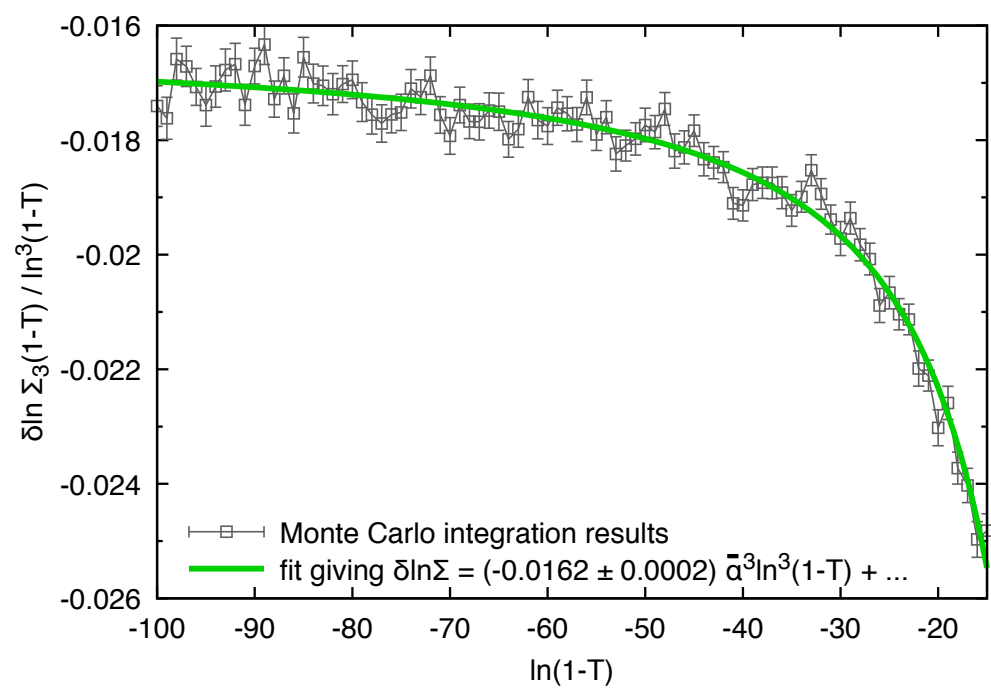

Figure 1. Evaluation of $\delta \ln \Sigma_{3}$ (defined here as the coefficient of $\bar{\alpha}^{3}$ rather than $\alpha_{s}^{3}$ as elsewhere in the text) for the thrust, $T$, as a function of $\ln (1-T)$, within the approximation of eqs. (B.7)-(B.12) of ref. [1], and divided by $\ln ^{3}(1-T)$ to help visualise the coefficient of $\bar{\alpha}^{3} \ln ^{3}(1-T)$. The fit function assumes a form for $\delta \ln \Sigma_{3}$ that is a third-order polynomial in powers of $\ln (1-T)$. The corresponding result for the coefficient of $\bar{\alpha}^{3} L^{3}$ in table 1 displays a larger, somewhat conservative error. This is to account for the dependence of the fit result on the precise choice of fit range, as well as potential correlations between points, which are not taken into account in the calculation of the error from the fit. 
Open Access. This article is distributed under the terms of the Creative Commons Attribution License (CC-BY 4.0), which permits any use, distribution and reproduction in any medium, provided the original author(s) and source are credited.

\section{References}

[1] M. Dasgupta, F.A. Dreyer, K. Hamilton, P.F. Monni and G.P. Salam, Logarithmic accuracy of parton showers: a fixed-order study, JHEP 09 (2018) 033 [arXiv: 1805.09327] [INSPIRE]. 